\title{
Pregnancy-associated breast cancer
}

\author{
Elena Cristina COCIRTA ${ }^{1}$, Nicolae GICA ${ }^{1,2}$, Radu BOTEZATU ${ }^{1,2}$, Gheorghe PELTECU ${ }^{1,2}$, \\ Anca Marina $\mathrm{CIOBANU}^{1}$, Corina $\mathrm{GICA}^{1}$, Anca Maria PANAITESCU ${ }^{1,2}$ \\ ${ }^{1}$ Filantropia Clinical Hospital, Bucharest, Romania \\ 2"Carol Davila" University of Medicine and Pharmacy, Bucharest, Romania
}

\section{ABStract}

Pregnancy-associated breast cancer is one of the leading pregnancy-related types of cancer with an increasing incidence. It represents a special situation which requires careful attention for the medical team in fulfilling the goals of the treatment: the cure of the disease and the well-being of the pregnancy. The decisions regarding the most appropriate therapeutic methods should be made by a multidisciplinary team after offering counseling to the patient. The diagnosis of breast cancer during pregnancy or breastfeeding is a challenging situation considering the physiological changes. The first line of treatment is considered to be surgery indicated in any trimester of pregnancy, with mastectomy being often used. Chemotherapy can be offered, except for the first trimester, taking into account the risk of malformations. Radiotherapy, hormonal and biological treatment are contraindicated during pregnancy. The fetus must be evaluated regularly during treatment and the decision of delivery should be made on obstetrical and oncological indications.
\end{abstract}

Keywords: breast cancer, pregnancy, oncological treatment, fetal surveillance

\section{INTRODUCTION}

Pregnancy-associated breast cancer (PABC) is defined as breast cancer diagnosed during pregnancy or in the first year postpartum. It represents a special and challenging situation for both the doctor and the patient, considering the need to treat a malignant pathology while taking into account the well-being of a fetus.

Neoplasia of the breast is the most common cancer diagnosed during pregnancy, representing up to $21 \%$ of all pregnancy-related malignancies [1]. The incidence of PABC is increasing, reaching 1:3,000 pregnancies, with more than $15 \%$ of women being under 35 years old and a median age at diagnosis of 33 years [2]. In the future the number of cases is expected to rise due to the fact that many women postpone childbearing.

Diagnosis of breast cancer can be more difficult in this subgroup, due to the changes associated with pregnancy and breastfeeding that can lead to delays of one to two months. The general practitioner and obstetrician are the firsts to whom the pregnant female presents $[3,4]$. Treatment for breast cancer during pregnancy has the same goals as in non-pregnant women, with curative intent and it depends on the trimester of pregnancy. Treatment options that can be used during pregnancy include surgery and systemic chemotherapy, while radiotherapy, hormonal therapy and immunotherapy are used only after delivery. Counseling should be offered and the decisions must be made by a multidisciplinary team with the consent of the patient. Maternal fetal medicine consultation and ultrasonographic assessment of the pregnancy with documentation of gestational age, fetal development and growth are necessary.

Pregnant women with breast cancer have a positive family history in $48 \%$ of case and in $9 \%$ of the cases there is an association with BRACA1 and BRACA2 
mutations [5]. Therefore, genetic testing and counseling are mandatory.

\section{SPECIFIC FEATURES OF BREAST CANCER DURING PREGNANCY}

Although the incidence of PABC is increasing, there are no screening programs, no preventive measures and no standardized diagnostic methods implemented in order to help health care workers in dealing with this illness.

Pregnancy associated physiological changes can promote breast cancer development. The hormonal profile of a pregnant woman, high levels of estrogen, progesterone and growth factors, are essential for fetal development, but they are also inducing tumor progression and transformation from pre-invasive to invasive lesions $[6,7]$.

Maternal immune tolerance and response are altered during pregnancy in order to increase the tolerance for the allogenic tissue that is represented by the growing fetus. In this case, malignant cells can easily escape immune system clearance and can further proliferate [8]. In addition, the pro-inflammatory environment from postpartum and post-lactation periods and the regression of the mammary gland, are also related to PABC process [9].

\section{DIAGNOSIS}

\section{Clinical examination}

Women often present with a palpable mass or skin changes. Due to the physiological changes in breast tissue during pregnancy and breastfeeding, such as hypertrophy, colostrum secretion, the diagnosis can be delayed [10]. Thus, it is important to have a record of the breast examination before pregnancy and to perform palpation of the breasts at the beginning of each trimester in cases with risk factors. Although $80 \%$ of palpable masses are benign (cyst, fibroadenoma, lipoma, galactocel, abscess), a new growing lesion which persists for more than 2 weeks should be investigated.

\section{Imaging examination}

The preferred method used to evaluate a breast mass during pregnancy is ultrasonography (USG). Its use is considered to be safe in all periods of a pregnancy and also postpartum [1]; in addition, it can help describe the features of a tumor raising concerns about its nature and it is useful to guide the needle when performing a biopsy. Breast USG has a high specificity and sensitivity helping to make distinction between a solid and a cystic mass [11].

Mammography (MMG) can also be used for the evaluation of the extension of the disease, the evalua- tion of the contralateral breast or in detecting focal asymmetry or microcalcifications [1]. Mammography should be used with abdominal shielding, although the radiation dose for the fetus is minimal [12]. It is recommended to start with an oblique view and if a suspicious image is detected, then both standard views will be taken [13].

The limitations of MMG consists in low accuracy due to the increased water content during pregnancy and fat distribution in the breast of young women; its sensitivity is approximately $86 \%$ [14].

Magnetic resonance imaging (MRI) is used when the results obtained with other methods are unclear, as MRI is considered to be safe in any trimester of pregnancy [15].

The American Academy of Pediatrics recommends caution in using MRI during the first trimester of pregnancy because the exposure during this period of pregnancy is not fully known [16]. The use of gadolinium in order to increase specificity and sensitivity was recommended, but there are controversies related to the fact that it crosses the placenta and could reach high fetal concentration with a potential impairment of fetal kidneys [17].

The use of gadolinium is indicated only in specific cases, although no side effects on the development of a fetus have been demonstrated [18].

Other radiological investigations should be made only in case of high clinical suspicion of metastases; in such cases X-ray of the chest and liver ultrasound are recommended. For the detection of bone metastases $\mathrm{X}$-ray and/or MRI are indicated [19].

\section{Histological examination}

Histological examination of a breast lesion is the standard procedure for diagnosing and characterizing it. Core needle or excisional biopsy are the preferred methods used having a high sensitivity, 98\% [20]. Fine-needle aspiration cytology (FNAC) has advantages, like minimal invasion, rapidity, low cost, but has a lower sensitivity and it is not able to provide additional information about cellular markers. Fine-needle aspiration cytology is useful to investigate the status of axillary lymph nodes. This information is important to decide between chemotherapy and surgery as primary treatment.

\section{Staging}

A complete staging using ionizing radiations is not always possible during pregnancy because of the risk of side effect to the fetus (organ malformations, mental retardation). So, the staging strategy should be individualized by the multidisciplinary team. Liver USG and chest radiography could be safely performed. 


\section{TREATMENT}

The treatment of PABC should be discussed within a multidisciplinary team formed by a medical oncologist, obstetrician, gynecology oncologist, pathologist, medical geneticist, fetal-maternal medicine specialist, neonatologist, radiotherapy specialist, psychologist. It is important for every patient to receive a personalized therapy after an individualized assessment of the case. The decisions regarding the course of treatment should be established with the patient's consent, after a thorough information over the risks and benefits. It is important not delaying the beginning of the treatment until delivery.

The purpose of the treatment is to cure the cancer, but in the same time two other goals need to be achieved: surveillance and support of the pregnancy and the well-being of the fetus.

\section{Surgical treatment}

Surgery for PABC is considered to be the first line of treatment, when is indicated and can be performed during any trimester of pregnancy [21]. Modified radical mastectomy is the treatment of choice and is preferred to breast conserving surgery, which requires radiotherapy (RT) [22].

During the first trimester, there is an insignificant risk for spontaneous abortion related to the general anesthesia [21]. Conservative surgery can be taken into consideration by the end of second and in the third trimester, with the use of adjuvant RT after birth. Breast reconstruction should be postponed after postpartum period to avoid prolonged anesthesia and additional risks and to avoid asymmetrical cosmetic effects due to pregnancy related modifications [19].

For patients with clinical positive lymph nodes, axillary dissection is recommended. In contrast, when discussing an early stage breast cancer, a high proportion of patients will have node-negative disease and could benefit from sentinel lymph node biopsy (SLNB) [23]. This procedure is demonstrated to have a lower morbidity when compared with axillary node dissection, with a shorter period for recovery $[24,25]$.

During pregnancy SLNB is a safe procedure. Although fetal radiation exposure can be associated with adverse effects like malformations, cancer or mental impairment, lymphoscintigraphy is safely applied during pregnancy when performing a low-dose technique $[24,26]$. In contrast, the use of blue dye (methylene or isosulfan blue) is not recommended due to the risk of anaphylactic reaction and potential risks for teratogenicity $[25,27]$. International guidelines do not discourage SLNB during pregnancy to be performed in centers where this is a routine procedure in non-pregnant women. However, they do not recommend the use of blue dye $[19,28]$.

\section{Systemic chemotherapy}

Chemotherapy (CTh) in PABC is indicated either as a neoadjuvant treatment in locally advanced breast cancer or as an adjuvant therapy when negative prognostic factors are present. The use of this type of agents should not be delayed, except when the cancer is diagnosed in the first trimester. When used as a postoperative treatment, it should be started within 3 weeks after surgery [29].

The use of CTh in the first trimester of pregnancy is contraindicated due to the concern of fetal teratogenicity [18]. The most vulnerable period for the fetus, concerning spontaneous abortion and malformations, is between 10 days to 8 weeks after conception, but CTh is contraindicated before the competition of the $14^{\text {th }}$ week's gestation [30].

Chemotherapy is considered to be safe when used in the second and third trimester of pregnancy. However, it is indicated to be ceased after 35 weeks of gestation or 3 weeks before delivery to enable the recovery of maternal bone marrow considering the risk of sepsis and hemorrhage in both the mother and the newborn $[19,29]$.

Administration of systemic chemotherapy during pregnancy is associated with an increased risk of intrauterine growth restriction and preterm delivery, the incidence varying between $5 \%$ and $8 \%[29,30]$. The risk of fetal malformation linked with the use of CTh during the second and third trimester is similar to the general population [31]. While on CTh, breastfeeding is not recommended because these agents pass into the breast milk and can induce neonatal leucopenia and an increased risk of infection. Breastfeeding should be started after at least 14 days after the last chemotherapy session $[12,19]$. Otherwise if the patient have to continue CTh after delivery milk production must be inhibited.

The most frequently used regimen of CTh during pregnancy is based on anthracycline, with a good safety profile [28]. The cardiac function of the patient needs to be evaluated using echocardiogram, before initiating treatment. Data have shown no increased risk for fetal cardiotoxicity following exposure to a regimen based on anthracycline $[32,33]$.

Taxanes represent an alternative treatment choice, not so widely studied, but with good outcomes and a good tolerability for the fetus [34]. It has been proven that taxanes are substrates of the P-glycoprotein that is highly expressed in the maternal part of the placenta, reducing the transplacental transfer of the taxanes and protecting the fetus $[30,34]$. This regimen should be used when indicated (node-positive or metastatic disease) or when anthracyclines are contraindicated $[19,28]$. 


\section{Radiotherapy}

Radiotherapy is contraindicated during pregnancy and is postponed until after delivery [19]. Exposure of the fetus to ionizing radiations increase the risk of miscarriage, teratogenicity, fetal growth restriction, mental retardation and childhood malignancies $[1,12]$. The effects depend on the dosage, radiation field extension and gestational age [26]. Delaying radiotherapy for more than 3 months in the treatment of PABC, can be associated with an increased risk of local recurrence and a poor prognosis [35].

\section{Hormonal treatment}

The use of Tamoxifen during any trimester of pregnancy is contraindicated. It should be used only after delivery, because of its association with fetal malformations (cranio-facial and genital) [26]. Also, women should not breastfeed when taking hormonal treatment because of the unknown transmission of the drug into the breast milk [19].

\section{Biological treatment}

Trastuzumab is a monoclonal antibody targeting the human epidermal growth factor receptor 2 (HER2 ) on the cell surface. It is highly expressed on the fetal kidney and interferes with amniotic fluid production leading to oligo- or anhydramnios during the second and third trimester when the transplacental transfer is increased [30]. The incidence of the amniotic fluid reduction increases with the treatment duration, reaching $61 \%$, but the effect is reversible when the administration of the drug is stopped [1]. Therefore, because of fetal adverse effects, administration of Trastuzumab should be postponed until after delivery $[19,28]$.

Other targeted therapies refer to vascular endothelial growth factor (VEGF) antagonist, as Bevacizumab, or small-molecule tyrosine-kinase inhibitors. As an antiangiogenetic agent, Bevacizumab has a very complex action endangering the embryonic development. So, both these targeted therapies are not indicated in treating PABC [19].

\section{FETAL SURVEILLANCE AND TIMING OF DELIVERY}

Pregnancy is considered to be of a high risk when associated with breast cancer and, consequently, the fetal well-being should be assessed by regular antenatal visits. Fetal ultrasound, biophysical profile or nonstress testing are recommended to be used at every 3-4 weeks [22].

Delivery should be delayed until after 37 weeks of pregnancy when the fetus is estimated to be at full term, unless there are obstetric or oncological indications for an early birth $[19,22]$. Nevertheless, for patients receiving chemotherapy, delivery should occur with at least 3 weeks after the last session. The route of delivery should be chosen based on obstetrics indications and whenever possible vaginal birth is preferred [12].

\section{CONCLUSIONS}

Pregnancy-associated breast cancer is one of the leading causes of neoplasia during pregnancy with an increasing incidence. It represents a challenging disease which requires a complex treatment and surveillance made by a multidisciplinary team in accordance with the patient's options.

The diagnosis of PABC is made using the same tools as in non-pregnant women, but it is more difficult to evaluate a tumor while physiological changes occur in the breast during pregnancy and breastfeeding.

Surgery is considered to be the treatment of choice and can be performed during any trimester; radical mastectomy is usually performed considering that local excision of the tumor requires radiotherapy, which is contraindicated during pregnancy. Axillary dissection is necessary when lymph nodes are clinically positive or harbor metastases proven by FNAC, but sentinel lymph node biopsy is an option for node negative disease.

Systemic chemotherapy is not used during the first trimester because of the risk of malformations, but when indicated, can be administrated in the second and third trimester with the recommendation to be stopped at 35 weeks of gestation or 3 weeks before delivery in order to minimize the risk of infection and hemorrhage for both mother and fetus.

Radiotherapy, hormonal therapy and biological treatment are used only after delivery because of their adverse effects on the fetus.

Fetal assessment should be performed by evaluating possible malformations, monitoring the growth, amniotic fluid index and the well-being. Delivery is indicated at full term, whenever possible and the route of birth should be chosen following obstetrical indications.

The family doctor needs to cooperate with the obstetrician to detect and monitor high risk pregnancies. General practitioner needs to be the beneficiary of these information that will allow him/her to provide better health care services to each patient.

\section{Acknowledgement}

All authors equally contributed to the present paper. 


\section{REFERENCES}

1. Zubor P, Kubatka P, Kapustova I, et al. Current Approaches in the Clinical Management of Pregnancy-Associated Breast Cancer-Pros and Cons. EPMA J. 2018;9(3):257-270.

2. Polivka J, Altun I, Golubnitschaja O. Pregnancy-associated breast cancer: the risky status quo and new concepts of predictive medicine. EPMA J. 2018; 9(1):1-13.

3. Garcia-Manero M, Royo MP, Espinos J, et al. Pregnancy associated breast cancer. Eur J Surg Oncol. 2009;35(2):215-8.

4. Woo JC, Yu T, Hurd TC, et al. Breast cancer in pregnancy: a literature review. Arch Surg. 2003;138(1):91-8.

5. Loman N, Johannsson O, Kristoffersson U, et al. Family history of breast and ovarian cancer and BRACA1 and BRACA2 mutations in a population-based series of early-onset breast cancer. J Natl Cancer Inst. 2001;93(16):1215-23.

6. Yager JD, Davidson NE. Estrogen carcinogenesis in breast cancer. N Engl J Med. 2006;354(3):270-282.

7. Gupta PB, Proia D, Cingoz O, et al. Systemic stromal effects of estrogen promote the growth of estrogen receptornegative cancers. Cancer Res. 2007; 67(5):2062-2071.

8. Shakhar K, Valdimarsdottir HB, Bovbjerg $\mathrm{DH}$. Heightened risk of breast cancer following pregnancy: could lasting systemic immune alterations contribute? Cancer Epidemiol Biomark Prev. 2007; 16(6):1082-1086.

9. O'Brien J, Lyons T, Monks J, et al. Alternatively activated macrophages and collagen remodeling characterize the postpartum involuting mammary gland across species. Am J Pathol. 2010; 176(3):1241-1255.

10. Theriault R, Hahn K. Management of breast cancer in pregnancy. Curr Oncol Rep. 2007; 9:17-21.

11. Navrozoglu I, Vrekkoussis T, Kontostolis E, et al. Breas cancer during pregnancy: a mini-review. Eur J Surg Oncol. 2008; 34(8):837-43.

12. Yu HH, Cheung PS, Leung RC, et al. Current management of pregnancy- associated breast cancer. Hong Kong Med J. 2017;23:387-94.

13. Amant F, Deckers $S$, Van Calsteren $K$, et al. Breast cancer in pregnancy: Recommendations of an international consensus meeting. Eur J Cancer. 2010; 46(18):3158-68.

14. Ahn BY, Kim HH, Moon WK, et al. Pregnancy- and-lactation-associated breast cancer: mammographic and sonographic findings. J Ultrasound Med. 2003; 22(5):491-497.

15. Kanal E, Barkovich AJ, Bell C, et al. ACR blue ribbon panel on MR safety. ACR guidance document for safe MR practices: 2007. AJR Am J Roentgenol. 2007; 188(6):1447-1474.

16. Oto A, Ernst R, Jesse MK, et al. Magnetic resonance imaging of the chest, abdomen and pelvis in the evaluation of pregnant patients with neoplasms. Am J Perinatol. 2007;24(4):243-50.

17. Bellin MF, Webb JA, Van Der Molen AJ, et al. Safety of MR liver specific contrast media. Eur Radiol. 2005;15(8):1607-14.

18. Sundgren $P C$, Leander $P$. Is administration of gadolinium-based contrast media to pregnant women and small children justified? J Magn Reson Imaging. 2011; 34(4):750-757.

19. RCOG Green-top guideline No. 12 Pregnancy and breast cancer. UK royal college of obstetricians and Gynaecologists; 2011.

20. Mitra S, Dey P. Fine-needle aspiration and core biopsy in the diagnosis of breast lesions: a comparison and review of the literature. Cytojournal. 2016;13:18.

21. Molckovsky A, Madarnas Y. Breast cancer in pregnancy: a literature review. Breast Cancer Res Treat. 2008;108:333-338.

22. Keyser EA, Staat BC, Fausett MB, Shields AD. Pregnancy-Associated Breast Cancer. Rev Obstet Gynecol. 2012;5(2):94-99.

23. Veronesi U., Paganelli G., Viale G. et al., A randomized comparison of sentinel-node biopsy with routine axillary dissection in breast cancer. N Engl J Med. 2003; 349:546-553.

24. Gentilini O, Cremonesi M, Toesca A, et al. Sentinel lymph node biopsy in pregnant patients with breast cancer. Eur J Nucl Med Mol Imaging. 2010;37:78-93.

25. Gropper AB, Calvillo KZ, Dominici L, et al. Sentinel lymph node biopsy in pregnant women with breast cancer. Ann Surg Oncol. 2014;21:2506-11.

26. Gentilini $\mathrm{O}$, Cremonesi $\mathrm{M}$, Trifirò $\mathrm{G}$, et al. Safety of sentinel node biopsy in pregnant patients with breast cancer. Ann Oncol. 2004;15:1348-51.

27. Khera SY, Kiluk JV, Hasson DM, et al. Pregnancy-associated breast cancer patients can safely undergo lymphatic mapping. Breast J. 2008;14(3):250-254.

28. Peccatori FA, Azim HA Jr, Orecchia R, et al. Cancer, pregnancy and fertility: ESMO clinical practice guidelines for diagnosis, treatment and follow-up. Ann Oncol. 2013;24 Suppl 6:vi160-70.

29. Padmagirison R, Gajjar K, Spencer C. Management of breast cancer during pregnancy. Obstet Gynecol. 2010; 12:186-92

30. Azim HA Jr, Del Mastro L, Scargone G, Peccatori FA. Treatment of breast cancer during pregnancy: regimen selection, pregnancy monitoring and more... Breast. 2011;20:1-6.

31. Martin JA, Hamilton BE, Ventura SJ, et al. Births: final data for 2009. Natl Vital Stat Rep. 2011;60:1-70.

32. Hahn K.M., Johnson P.H., Gordon N., et al. Treatment of pregnant breast cancer patients and outcomes of children exposed to chemotherapy in utero. Cancer. 2006; 107:1219-1226.

33. Peccatori F.A., Azim Jr. H.A., Scarfone G., et al. Weekly epirubicin in the treatment of gestational breast cancer (GBC). Breast Cancer Treat. 2009;115: 591-594.

34. Zagouri F, Sergentanis TN, Chrysikos D, et al. Taxanes for breast cancer during pregnancy: a systematic review. Clin Breast Cancer. 2013;13(1):16-23.

35. Hershman DL, Wang X, McBride R, et al. Delay in initiating adjuvant radiotherapy following breast conservation surgery and its impact on survival. Int $J$ Radiat Oncol Biol Phys. 2006;65(5):1353-1360. 